\title{
A Mechanical Force Accompanies Fluorescence Resonance Energy Transfer (FRET)
}

\author{
Adam E. Cohen*,: and Shaul Mukamel ${ }^{\dagger}$ \\ Semiconductor Physics, Cavendish Laboratory, Cambridge University, Cambridge CB3 OHE, England, and \\ Departments of Chemistry and Physics and Astronomy, University of Rochester, Rochester, New York 14627
}

Received: October 24, 2002

\begin{abstract}
We show that the coupling responsible for fluorescence resonance energy transfer (FRET) generates a mechanical force that is distinct from the van der Waals force between ground-state molecules and can be either attractive or repulsive. The underlying potential has the same $1 / r^{6}$ distance dependence as the rate of FRET, and the two are connected by a Kramers-Kronig relation. Just as the rate of FRET can be derived either classically or quantum mechanically, so too can the interaction potential. Because of the FRET force, time-resolved FRET measurements contain information on the mechanical stiffness of the matrix containing donor and acceptor.
\end{abstract}

\section{Introduction}

Fluorescence resonance energy transfer (FRET) from an excited donor molecule to a nearby acceptor plays a major role in photosynthesis, carrying energy from chlorophyll molecules to the photosynthetic reaction center. ${ }^{1}$ FRET also provides a nanoscale ruler: when donor and acceptor are attached to a biomolecule, the rate of FRET indicates the donor-acceptor distance. ${ }^{2,3}$ There has recently been a resurgence of interest in FRET in connection with single-molecule studies as a probe for conformations of polymers and biomolecules. ${ }^{4}$

FRET and long-range dispersion forces both arise from a coupling between the transition dipoles of two molecules. Thus it is reasonable to expect a change in the long-range intermolecular force to accompany the process of FRET. This FRET force (FF) has implications both for photosynthesis and for biophysical FRET studies. A force accompanying photosynthetic FRET may lead to functionally significant conformational changes in the protein scaffold around chlorophyll molecules. Biophysical FRET studies usually assume that the FRET pair does not affect the molecule under study. This is justified because the FF tends to be weak, corresponding to an interaction free energy of $\sim 0.1 k_{\mathrm{B}} T$ at a donor-acceptor separation of 1 $\mathrm{nm}$. However, small changes in intermolecular force can have a macroscopic effect near a critical point where all other forces along a conformational coordinate vanish. Polymer solutions can be brought near a critical point by adjusting temperature and solvent composition. ${ }^{5}$ Moreover, the force creates the possibility to use light to control the conformation of a biomolecule and to probe its mechanical response.

One way to think about the FF is as a generalization of optical trapping. A polarizable particle (atom, molecule, colloid, etc.) experiences a force along an electric field gradient. In laser tweezers, tight focusing of the laser beam creates the field gradient. ${ }^{6}$ It has been proposed to use sharp metal tips to enhance optical fields and field gradients. ${ }^{7}$ Consider the limit in which the sharp metal tip is shrunk to a single molecule. The nearfield of an excited molecule has strong fields and strong field gradients. Thus the FF can be thought of as optical trapping of the acceptor by the near-field of the excited donor.

\footnotetext{
$\doteqdot$ Cambridge University

University of Rochester.
}

This electrodynamic picture can be formally established by using the multipolar Hamiltonian in which all intermolecular interactions are mediated by photons, so there is no explicit donor-acceptor coupling in the Hamiltonian. ${ }^{8}$ The Coulomb force is recovered in the near-field limit, where retardation is neglected. This picture can be obtained classically because the Hamiltonian for a harmonic oscillator is identical in classical and quantum mechanics. When the acceptor is modeled as a collection of harmonic oscillators and then this response is lumped into the complex polarizability function, $\alpha(\omega)$, the system appears to be completely classical. ${ }^{23,24}$

A second way to understand the FRET force is to consider the eigenstates of the donor and acceptor in the minimal coupling Hamiltonian with the Coulomb coupling included explicitly. ${ }^{9}$ In this picture, the force arises from a radiative shift in the energy levels of the donor induced by its coupling to the acceptor. The minimal coupling and multipolar Hamiltonians are related by a canonical gauge transformation and the two descriptions are both exact and equivalent, even though they offer completely different physical pictures. ${ }^{15,24}$

There have been many calculations of the van der Waals force between two two-level molecules with one molecule excited. ${ }^{10-12}$ These calculations show that optical excitation can dramatically alter the long-range force. However, it is not clear how or whether these formal calculations apply to real multilevel molecules in the presence of relaxation and dephasing nor what the relation is between the force and the energy transfer.

In this paper, we show how the force may be calculated from observable emission and absorption spectra. This is done using both the minimal coupling and the multipolar Hamiltonians.

\section{Electrodynamic Derivation}

A. Molecule in a Field. A spatially uniform electric field

$$
\mathbf{E}(t)=\hat{\mathbf{e}} E_{0} \sin \omega t
$$

induces an oscillating dipole moment, $\mathbf{p}_{\mathrm{A}}(t)$, in a molecule

$\mathbf{p}_{\mathrm{A}}(t)=\alpha_{\mathrm{A}}^{\prime} \hat{\mathbf{n}}_{\mathrm{A}}\left(\hat{\mathbf{n}}_{\mathrm{A}} \cdot \hat{\mathbf{e}}\right) E_{0} \sin (\omega t)-\alpha_{\mathrm{A}}^{\prime \prime} \hat{\mathbf{n}}_{\mathrm{A}}\left(\hat{\mathbf{n}}_{\mathrm{A}} \cdot \hat{\mathbf{e}}\right) E_{0} \cos (\omega t)$

where $\alpha_{\mathrm{A}}^{\prime}$ and $\alpha_{\mathrm{A}}^{\prime \prime}$ are the real and imaginary parts, respectively, of the frequency-dependent complex polarizability tensor, 
$\alpha_{\mathrm{A}} \equiv\left(\alpha_{\mathrm{A}}^{\prime}+i \alpha_{\mathrm{A}}^{\prime \prime}\right) \hat{\mathbf{n}}_{\mathrm{A}} \hat{\mathbf{n}}_{\mathrm{A}}$. The unit vector $\hat{\mathbf{n}}_{\mathrm{A}}$ lies along the principle absorption dipole of the molecule.

The molecule absorbs power $P$ :

$$
\begin{aligned}
P & =\left\langle\mathbf{E} \cdot \dot{\mathbf{p}}_{\mathrm{A}}\right\rangle_{t} \\
& =\frac{1}{2} E_{0}{ }^{2}\left|\hat{\mathbf{n}}_{\mathrm{A}} \cdot \hat{\mathbf{e}}\right|^{2} \omega \alpha_{\mathrm{A}}^{\prime \prime}(\omega)
\end{aligned}
$$

where the average is taken over many optical cycles. If the electric field has a power spectral density, $f(\omega)$, where $\int f(\omega)$ $\mathrm{d} \omega=1$, then the molecule absorbs a power per unit frequency

$$
P(\omega)=\frac{1}{2} E_{0}{ }^{2}\left|\hat{\mathbf{n}}_{\mathrm{A}} \cdot \hat{\mathbf{e}}\right|^{2} \omega \alpha_{\mathrm{A}}^{\prime \prime}(\omega) f(\omega)
$$

and the total power absorbed is $P=\int_{0}^{\infty} P(\omega) \mathrm{d} \omega$.

The molecule also experiences a change in its mean free energy, $U$ :

$$
\begin{aligned}
U & =-\frac{1}{2}\left\langle\mathbf{E} \cdot \mathbf{p}_{\mathrm{A}}\right\rangle_{t} \\
& =-\frac{1}{4} E_{0}{ }^{2}\left|\hat{\mathbf{n}}_{\mathrm{A}} \cdot \hat{\mathbf{e}}\right|^{2} \alpha_{\mathrm{A}}^{\prime}(\omega)
\end{aligned}
$$

If the field has a power spectral density $f(\omega)$, then the change in free energy per unit frequency is

$$
U(\omega)=-\frac{1}{4} E_{0}^{2}\left|\hat{\mathbf{n}}_{\mathrm{A}} \cdot \hat{\mathbf{e}}\right|^{2} \alpha_{\mathrm{A}}^{\prime}(\omega) f(\omega)
$$

and the total energy shift is $U=\int_{0}^{\infty} U(\omega) \mathrm{d} \omega$.

Comparison of eqs 4 and 6 shows that the change in free energy and rate of excitation (both per unit frequency) are related:

$$
U(\omega)=-\frac{1}{2}\left(\frac{\alpha_{\mathrm{A}}^{\prime}(\omega)}{\alpha_{\mathrm{A}}^{\prime \prime}(\omega)}\right) \hbar K(\omega)
$$

where we have replaced the power absorbed by the rate of excitation, $K(\omega)=P(\omega) / \hbar \omega$. This equation is the basis for all of the results of our paper.

Field gradients cause the rate of excitation, and hence the free energy, to depend on molecular position, r. Molecular anisotropy causes the rate of excitation to depend on the angle, $\theta$, between the molecular axis and the local field. Making note of these effects, we get a position- and orientation-dependent free energy, $U(\mathbf{r}, \theta)$, which leads to a force $\mathbf{F}=-\nabla U(\mathbf{r}, \theta)$ and to a torque $\tau=-\partial U(\mathbf{r}, \theta) / \partial \theta$.

The electric field, $\mathbf{E}(t)$, could come from a light source, or it could be produced by a nearby excited donor molecule: the acceptor, $A$, responds the same way regardless of the source of the field. When the electric field comes from laser light, the $\mathbf{r}$ and $\theta$ dependence of $U(\mathbf{r}, \theta)$ lead to optical trapping ${ }^{6}$ and the optical Kerr effect, respectively. ${ }^{13}$ In this case, the $\mathbf{r}$ dependence comes from the tight focusing of the laser beam. When the electric field comes from an excited donor molecule, the spatial dependence originates from the near-field variation of the field created by the donor, and eq 7 gives the intermolecular potential associated with FRET. In principle, there is also a torque that seeks to align anisotropic molecules participating in FRET. This torque will not be considered here.
B. Application to FRET. Forster showed that the rate of FRET is ${ }^{26}$

$$
k_{\text {FRET }}=\frac{9 c^{4} \kappa^{2}}{8 \pi \tau_{\mathrm{D}} r^{6}} \int_{0}^{\infty} \frac{f_{\mathrm{D}}(\omega) \sigma_{\mathrm{A}}(\omega)}{n^{4}(\omega) \omega^{4}} \mathrm{~d} \omega
$$

where $\tau_{\mathrm{D}}$ is the lifetime of the donor, $r$ is the distance between donor and acceptor, $f_{\mathrm{D}}(\omega)$ is the normalized emission spectrum of the donor, $\sigma_{\mathrm{A}}(\omega)$ is the absorption cross section of the acceptor, and $n(\omega)$ is the refractive index of the medium surrounding the donor and acceptor. ${ }^{14}$ The factor $\kappa \equiv$ $3\left(\hat{\mathbf{n}}_{\mathrm{A}} \cdot \hat{\mathbf{r}}\right)\left(\hat{\mathbf{n}}_{\mathrm{D}} \cdot \hat{\mathbf{r}}\right)-\hat{\mathbf{n}}_{\mathrm{A}} \cdot \hat{\mathbf{n}}_{\mathrm{D}}$ takes into account the relative orientation of donor and acceptor transition dipoles. To express $k_{\mathrm{FRET}}$ in terms of the polarizability, we make the substitution

$$
\sigma_{\mathrm{A}}(\omega)=\frac{\omega \alpha_{\mathrm{A}}^{\prime \prime}(\omega)}{3 c \epsilon_{0} n(\omega)}
$$

so that

$$
k_{\mathrm{FRET}}=\int_{0}^{\infty} \frac{3 c^{3} \kappa^{2}}{8 \pi \epsilon_{0} \tau_{\mathrm{D}} r^{6}} \frac{f_{\mathrm{D}}(\omega) \alpha_{\mathrm{A}}^{\prime \prime}(\omega)}{n^{5}(\omega) \omega^{3}} \mathrm{~d} \omega
$$

The integrand of eq 10 is precisely the $K(\omega)$ in eq 7. Combining eqs 7 and 10, we find the interaction free energy associated with FRET

$$
U_{\mathrm{FRET}}=-\frac{3 \hbar c^{3} \kappa^{2}}{16 \pi \epsilon_{0} \tau_{\mathrm{D}} r^{6}} \int_{0}^{\infty} \frac{f_{\mathrm{D}}(\omega) \alpha_{\mathrm{A}}^{\prime}(\omega)}{n^{5}(\omega) \omega^{3}} \mathrm{~d} \omega
$$

C. Kramers-Kronig Relations. Both the real and imaginary parts of the molecular polarizability can be readily computed for models and at various levels of theory. Absorption experiments give the imaginary part, but the real part is harder to measure. To eliminate $\alpha^{\prime}(\omega)$ in the expression for $U_{\text {FRET }}$, we use the Kramers-Kronig formula:

$$
\alpha_{\mathrm{A}}^{\prime}(\omega)=\frac{2}{\pi} \mathscr{P} \int_{0}^{\infty} \frac{\omega^{\prime} \alpha_{\mathrm{A}}^{\prime \prime}\left(\omega^{\prime}\right)}{\omega^{\prime 2}-\omega^{2}} \mathrm{~d} \omega^{\prime}
$$

where $\mathscr{D}$ indicates the principal value of the integral. Thus

$$
U_{\text {FRET }}=-\frac{3 \hbar c^{3} \kappa^{2}}{16 \pi \epsilon_{0} \tau_{\mathrm{D}} r^{6}} \int_{0}^{\infty} \frac{2}{\pi} \mathscr{\mathscr { P }} \int_{0}^{\infty} \frac{f_{\mathrm{D}}(\omega) \omega^{\prime} \alpha_{\mathrm{A}}^{\prime \prime}\left(\omega^{\prime}\right)}{n^{5}(\omega) \omega^{3}\left(\omega^{\prime 2}-\omega^{2}\right)} \mathrm{d} \omega^{\prime} \mathrm{d} \omega
$$

This expression almost contains $K(\omega)$, except that $f_{\mathrm{D}}(\omega)$ and $\alpha^{\prime \prime}\left(\omega^{\prime}\right)$ occur at different frequencies. We thus define a new quantity, $K\left(\omega, \omega^{\prime}\right)$ :

$$
K\left(\omega, \omega^{\prime}\right) \equiv \frac{3 c^{3} \kappa^{2}}{8 \pi \epsilon_{0} \tau_{\mathrm{D}} r^{6}} \frac{f_{\mathrm{D}}(\omega) \alpha_{\mathrm{A}}^{\prime \prime}\left(\omega^{\prime}\right)}{n^{5}(\omega) \omega^{3}}
$$

This two-dimensional transition density corresponds to the rate of FRET that would occur if the spectrum of the acceptor were shifted along the frequency axis relative to the spectrum of the donor. While this shift cannot be easily realized experimentally (i.e., it is hard to make a family of molecules with shifted spectra but the same spectral line shape), it is easy to compute $K\left(\omega, \omega^{\prime}\right)$ from a known donor emission spectrum and acceptor absorption spectrum. In terms of $K\left(\omega, \omega^{\prime}\right)$,

$$
k_{\mathrm{FRET}}=\int_{0}^{\infty} \int_{0}^{\infty} K\left(\omega, \omega^{\prime}\right) \delta\left(\omega^{\prime}-\omega\right) \mathrm{d} \omega^{\prime} \mathrm{d} \omega
$$


and

$$
U_{\text {FRET }}=-\frac{\hbar}{\pi} \int_{0}^{\infty} \mathscr{P} \int_{0}^{\infty} \frac{\omega^{\prime} K\left(\omega, \omega^{\prime}\right)}{\omega^{\prime 2}-\omega^{2}} \mathrm{~d} \omega^{\prime} \mathrm{d} \omega
$$

\section{Eigenstate Derivation}

In the previous section, we considered a molecule with arbitrary polarizability, $\alpha(\omega)$, and focused on how $\alpha(\omega)$ mediates the response to an electric field. This corresponds to the multipolar Hamiltonian. In the alternative approach, based on the minimal coupling Hamiltonian, we consider the internal eigenstates of the donor and acceptor molecules and include the dipole-dipole coupling as a perturbation to these states. Then the question is how does the coupling affect the expectation value of the energy? This calculation closely follows Förster's original paper on FRET $^{22}$ and yields the same results as the previous calculation.

In the Wigner-Weiskopff model, when a state, $i$, is coupled to a broad continuum, $\{j\}$, an effective Hamiltonian arises that adds a self-energy to the energy of the isolated state: $E_{i} \rightarrow E_{i}$ $+R_{i \cdot}{ }^{15}$ Here

$$
R_{i}=\lim _{\eta \rightarrow 0^{+}} \sum_{j} \frac{\left|V_{i j}\right|^{2}}{E_{i}-E_{j}+\imath \eta}
$$

where $V_{i j}$ is the matrix element coupling states $i$ and $j$ and $E_{i}$ and $E_{j}$ are the energies of these states. This self-energy is partitioned as

$$
R_{i}=U_{i}-\frac{i \hbar}{2} k_{i}
$$

where the real part, $U_{i}$, gives the level shift of state $i$, and the imaginary part, $k_{i}$, gives the rate of decay of state $i$. The Kramers-Kronig relation between the two follows immediately from the analytic properties of the self-energy, which are a direct consequence of causality.

Let the initial state, $i$, correspond to the excited donor and ground-state acceptor. The manifold of final states, $\{j\}$, corresponds to the ground-state donor and excited acceptor (we assume that the acceptor has densely distributed levels). Replacing the sum in eq 17 by an integral over acceptor states, we find that the rate of energy transfer is given by Fermi's golden rule

$$
k_{i}=\frac{2 \pi}{\hbar} \int\left|V_{i j}\right|^{2} \rho\left(E_{j}\right) \delta\left(E_{i}-E_{j}\right) \mathrm{d} E_{j}
$$

where $\rho\left(E_{j}\right)$ is density of transitions of energy $E_{j}$.

The coupling to a continuum also produces an energy shift, $U_{i}$, of the initial state:

$$
U_{i}=\mathscr{P} \int \frac{\left|V_{i j}\right|^{2} \rho\left(E_{j}\right)}{E_{i}-E_{j}} \mathrm{~d} E_{\mathrm{j}}
$$

Förster used eq 19 to calculate the rate of FRET. We follow his procedure but apply it to eq 20 to calculate the energy shift.

The wave functions of the donor-acceptor system in its initial and final states are

$$
\begin{aligned}
& \Psi_{i}=\phi_{\mathrm{D}}^{*} \phi_{\mathrm{A}} \Phi_{\mathrm{D}}^{*} \Phi_{\mathrm{A}} \\
& \Psi_{j}=\phi_{\mathrm{D}} \phi_{\mathrm{A}}^{*} \Phi_{\mathrm{D}} \Phi_{\mathrm{A}}^{*}
\end{aligned}
$$

where $\phi_{\mathrm{A}}$ and $\phi_{\mathrm{D}}$ are the electronic wave functions and $\Phi_{\mathrm{A}}$ and
$\Phi_{\mathrm{D}}$ are the nuclear wave functions. The matrix element for the transition is

$$
\begin{aligned}
V_{i j} & =\left\langle\Psi_{j}\left|\frac{e^{2}}{4 \pi \epsilon_{0} n^{2} \mathbf{r}}\right| \Psi_{i}\right\rangle \\
& =V_{\mathrm{e}}\left\langle\Phi_{\mathrm{D}} \mid \Phi_{\mathrm{D}}^{*}\right\rangle\left\langle\Phi_{\mathrm{A}}^{*} \mid \Phi_{\mathrm{A}}\right\rangle
\end{aligned}
$$

The nuclear components of $V_{i j}$ are the Franck-Condon factors $S_{\mathrm{D}}\left(E_{\mathrm{D}}^{*}, E_{\mathrm{D}}\right) \equiv\left\langle\Phi_{\mathrm{D}} \mid \Phi_{\mathrm{D}}^{*}\right\rangle$ and $S_{\mathrm{A}}\left(E_{\mathrm{A}}, E_{\mathrm{A}}^{*}\right) \equiv\left\langle\Phi_{\mathrm{A}}^{*} \mid \Phi_{\mathrm{A}}\right\rangle$.

There are many ways to arrive at the electronic component of the matrix element, $V_{\mathrm{e}} \equiv\left\langle\phi_{\mathrm{D}} \phi_{\mathrm{A}}^{*}\left|e^{2} /\left(4 \pi \epsilon_{0} n^{2} \mathbf{r}\right)\right| \phi_{\mathrm{D}}^{*} \phi_{\mathrm{A}}\right\rangle$. Note that to calculate forces it is necessary to know the gradient in $V_{\mathrm{e}}$ at the equilibrium donor-acceptor separation. If the molecules are neutral and their separation is large compared to their size, then the point dipole approximation is appropriate:

$$
V_{\mathrm{e}}=\frac{\mu_{\mathrm{A}} \mu_{\mathrm{D}} \kappa}{4 \pi \epsilon_{0} n^{2} r^{3}}
$$

For molecules for which the separation is small compared to the size but there is still negligible electronic overlap (e.g., chlorophyll molecules in the bacterial photosynthetic antenna complex), then the point dipole approximation breaks down and $V_{\mathrm{e}}$ must be calculated numerically. ${ }^{16}$ Standard quantum chemistry codes exist to do this at varying levels of theory. The coupling can also be extracted from spectroscopic data: the Davydov splitting in the donor-acceptor pair is twice the electronic coupling energy. ${ }^{17}$ It may be possible to obtain the gradient of $V_{\mathrm{e}}$ from the pressure dependence of the Davydov splitting.

Typically the donor in its excited state occupies a distribution of vibrational energy levels with probability $g_{\mathrm{D}}\left(E_{\mathrm{D}}^{*}\right)$. Similarly, the acceptor in its ground-state occupies a distribution of vibrational energy levels with probability $g_{\mathrm{A}}\left(E_{\mathrm{A}}\right)$. The total rate of FRET is given by the rate for each microstate $\left(E_{\mathrm{D}}^{*}, E_{\mathrm{A}}\right)$ weighted by the probability for the system to be in that microstate and summed over all microstates. If $g_{\mathrm{D}}\left(E_{\mathrm{D}}^{*}\right)$ and $g_{\mathrm{A}}\left(E_{\mathrm{A}}\right)$ are statistically independent (i.e., they arise from different vibrational modes), then this sum factors into two components. One component depends only on the emission spectrum of the donor; the other depends only on the absorption spectrum of the acceptor. We apply this procedure to compute the energy shift.

From eq 20, the total energy shift for all initial states of the donor is

$$
U_{\text {FRET }}=\int \mathscr{Q} \int \frac{\rho\left(E_{i}\right)\left|V_{i j}\right|^{2} \rho\left(E_{j}\right)}{E_{i}-E_{j}} \mathrm{~d} E_{j} \mathrm{~d} E_{i}
$$

The numerator of the integrand of eq 24 can be expanded to yield

$$
\begin{aligned}
& \rho\left(E_{i}\right)\left|V_{i j}\right|^{2} \rho\left(E_{j}\right)= \\
& \left|V_{\mathrm{e}}\right|^{2}\left(\int g_{\mathrm{D}}\left(E_{\mathrm{D}}^{*}\right) S_{\mathrm{D}}{ }^{2}\left(E_{\mathrm{D}}^{*}, E_{\mathrm{D}}^{*}-E_{i}\right) \mathrm{d} E_{\mathrm{D}}^{*}\right)\left(\int g _ { \mathrm { A } } ( E _ { \mathrm { A } } ) S _ { \mathrm { A } } { } ^ { 2 } \left(E_{\mathrm{A}}, E_{\mathrm{A}}+\right.\right. \\
& \left.\left.E_{j}\right) \mathrm{~d} E_{\mathrm{A}}\right)
\end{aligned}
$$

which contains separate integrals over the donor and acceptor coordinates. Each integral gives an observable. The donor 
integral is related to the normalized donor emission spectrum, $f_{\mathrm{D}}\left(\omega_{i}\right)$ (measured in photons per unit frequency), via ${ }^{18}$

$$
\int g_{\mathrm{D}}\left(E_{\mathrm{D}}^{*}\right) S_{\mathrm{D}}^{2}\left(E_{\mathrm{D}}^{*}, E_{\mathrm{D}}^{*}-E_{i}\right) \mathrm{d} E_{\mathrm{D}}^{*}=\frac{3 \pi \epsilon_{0} \hbar c^{3}}{\omega_{i}^{3} n\left(\omega_{i}\right) \mu_{\mathrm{D}}{ }^{2} \tau_{\mathrm{D}}} f_{\mathrm{D}}\left(\omega_{i}\right)
$$

The acceptor integral is related to the acceptor absorption cross section, $\sigma_{\mathrm{A}}\left(\omega_{j}\right)$, via

$$
\int g_{\mathrm{A}}\left(E_{\mathrm{A}}\right) S_{\mathrm{A}}^{2}\left(E_{\mathrm{A}}, E_{\mathrm{A}}+E_{j}\right) \mathrm{d} E_{\mathrm{A}}=\frac{3 \epsilon_{0} \hbar c n\left(\omega_{j}\right)}{\pi \omega_{j} \mu_{\mathrm{A}}^{2}} \sigma_{\mathrm{A}}\left(\omega_{j}\right)
$$

If we assume that the index of refraction is weakly dependent on frequency, then $n\left(\omega_{j}\right) / n\left(\omega_{i}\right)$ cancels in the product of eqs 26 and 27. Combining eqs 24-27 and converting to integrals over frequency gives $U_{\text {FRET }}$ in terms of experimentally accessible parameters:

$$
U_{\mathrm{FRET}}=-\frac{\left|V_{\mathrm{e}}\right|^{2} 9 \hbar \epsilon_{0}{ }^{2} c^{4}}{\mu_{\mathrm{A}}{ }^{2} \mu_{\mathrm{D}}{ }^{2} \tau_{\mathrm{D}}} \int \mathscr{P} \int \frac{f_{\mathrm{D}}\left(\omega_{i}\right) \sigma_{\mathrm{A}}\left(\omega_{j}\right)}{\omega_{i}^{3} \omega_{j}\left(\omega_{j}-\omega_{i}\right)} \mathrm{d} \omega_{j} \mathrm{~d} \omega_{i}
$$

Substituting $\alpha^{\prime \prime}\left(\omega_{j}\right)$ for $\sigma\left(\omega_{j}\right)$ (eq 9) gives

$$
U_{\mathrm{FRET}}=-\frac{\left|V_{\mathrm{e}}\right|^{2} 3 \hbar \epsilon_{0} c^{3}}{\mu_{\mathrm{A}}{ }^{2} \mu_{\mathrm{D}}{ }^{2} \tau_{\mathrm{D}}} \int \mathscr{P} \int \frac{f_{\mathrm{D}}\left(\omega_{i}\right)}{n\left(\omega_{j}\right) \omega_{i}{ }^{3}} \frac{\alpha_{\mathrm{A}}^{\prime \prime}\left(\omega_{j}\right)}{\omega_{j}-\omega_{i}} \mathrm{~d} \omega_{j} \mathrm{~d} \omega_{i}
$$

Equation 29 contains a Kramers-Kronig relation for $\alpha_{A}^{\prime}\left(\omega_{i}\right)$, so making this substitution and inserting the point dipole approximation for $V_{\mathrm{e}}$ (eq 23) yields

$$
U_{\mathrm{FRET}}=-\frac{3 \hbar c^{3}}{16 \pi \epsilon_{0} n^{5} r^{6} \tau_{\mathrm{D}}} \int_{0}^{\infty} \frac{f_{\mathrm{D}}(\omega) \alpha_{\mathrm{A}}^{\prime}(\omega)}{\omega^{3}} \mathrm{~d} \omega
$$

which is exactly the same as the classically derived eq 11 .

\section{Examples}

A. Lorentzian Line Shapes. The Lorentzian line shape describes the response of a damped harmonic oscillator (classical or quantum mechanical) and also the linear response of a quantum mechanical two-level system. Consider the case where both the acceptor and donor are characterized by a Lorentzian response with resonant frequencies $\omega_{\mathrm{A}}$ and $\omega_{\mathrm{D}}$, respectively:

$$
\begin{aligned}
& \alpha_{\mathrm{A}}^{\prime}(\omega)=\frac{\mu_{\mathrm{A}}{ }^{2}}{\hbar} \frac{\omega_{\mathrm{A}}-\omega}{\left(\omega_{\mathrm{A}}-\omega\right)^{2}+\gamma_{\mathrm{A}}^{2}} \\
& \alpha_{\mathrm{A}}^{\prime \prime}(\omega)=\frac{\mu_{\mathrm{A}}^{2}}{\hbar} \frac{\gamma_{\mathrm{A}}}{\left(\omega_{\mathrm{A}}-\omega\right)^{2}+\gamma_{\mathrm{A}}^{2}}
\end{aligned}
$$

and

$$
f_{\mathrm{D}}(\omega)=\frac{\gamma_{\mathrm{D}} / \pi}{\left(\omega_{\mathrm{D}}-\omega\right)^{2}+\gamma_{\mathrm{D}}^{2}}
$$

Further assume that the index of refraction is weakly dependent on frequency in the region of interest and the spectra are narrow enough to replace $1 / \omega^{3}$ by $1 / \omega_{0}^{3}$, where $\omega_{0} \approx\left(\omega_{\mathrm{A}}+\omega_{\mathrm{D}}\right) / 2$. The integrals in eqs 10 and 11 then evaluate to

$$
k_{\mathrm{FRET}}=\frac{3 c^{3} \mu_{\mathrm{A}}{ }^{2} \kappa^{2}}{8 \pi \epsilon_{0} \tau_{\mathrm{D}} n^{5} \hbar \omega_{0}{ }^{3} r^{6}} \frac{\gamma_{\mathrm{A}}+\gamma_{\mathrm{D}}}{\left(\omega_{\mathrm{A}}-\omega_{\mathrm{D}}\right)^{2}+\left(\gamma_{\mathrm{A}}+\gamma_{\mathrm{D}}\right)^{2}}
$$

and

$$
\begin{aligned}
U_{\mathrm{FRET}} & =\frac{3 c^{3} \mu_{\mathrm{A}}{ }^{2} \kappa^{2}}{16 \pi \epsilon_{0} \tau_{\mathrm{D}} n^{5} \omega_{0}{ }^{3} r^{6}} \frac{\omega_{\mathrm{D}}-\omega_{\mathrm{A}}}{\left(\omega_{\mathrm{A}}-\omega_{\mathrm{D}}\right)^{2}+\left(\gamma_{\mathrm{A}}+\gamma_{\mathrm{D}}\right)^{2}} \\
& =\frac{\omega_{\mathrm{D}}-\omega_{\mathrm{A}}}{2\left(\gamma_{\mathrm{D}}+\gamma_{\mathrm{A}}\right)} \hbar k_{\mathrm{FRET}}
\end{aligned}
$$

Equation 34 shows that $U_{\text {FRET }}$ vanishes on resonance $\left(\omega_{\mathrm{D}}=\right.$ $\left.\omega_{\mathrm{A}}\right)$ and in the absence of spectral overlap $\left(\left|\omega_{\mathrm{D}}-\omega_{\mathrm{A}}\right| \gg \gamma_{\mathrm{D}}+\right.$ $\left.\gamma_{\mathrm{A}}\right)$. It is maximized for $\omega_{\mathrm{D}}-\omega_{\mathrm{A}}= \pm\left(\gamma_{\mathrm{D}}+\gamma_{\mathrm{A}}\right)$. If $\omega_{\mathrm{D}}>\omega_{\mathrm{A}}$, then $U_{\text {FRET }}$ is positive and the force is repulsive.

There is a simple intuitive explanation for the dependence of the sign of the force on the sign of $\omega_{\mathrm{D}}-\omega_{\mathrm{A}}$. Picture the oscillating field from the donor driving the polarization of the acceptor. If the driving frequency is below the resonant frequency of the acceptor, then the polarization of the acceptor is in-phase with the driving field because the acceptor can respond essentially instantaneously to the field. This leads to a negative energy or an attractive force. However, if the driving frequency is greater than the resonant frequency of the acceptor, then the acceptor is never able to "catch up" with the quickly varying driving field. The dipole moment of the acceptor acquires an out of phase component, which points in the wrong direction, and the energy is positive or repulsive. For homotransfer, the FF is always attractive because the Stokes shift guarantees that $\omega_{\mathrm{D}}<\omega_{\mathrm{A}}$.

Line shapes are usually Lorentzian near the center but have much shorter wings (and finite second and higher moments). In many cases, the wings are Gaussian. The Voigt profile (convolution of a Gaussian and a Lorentzian) or the stochastic model of Kubo are commonly used models that interpolate between the two profiles. ${ }^{15,19}$ Provided the detuning, $\omega_{\mathrm{D}}-\omega_{\mathrm{A}}$, is small enough for the line shapes to overlap in the Lorentzian regime, then eq 34 provides a simple way to estimate the force accompanying FRET.

B. Sample Calculation. The FF can easily be calculated from experimental spectra via the relation

$$
U_{\mathrm{FRET}}=\frac{-\int f_{\mathrm{D}}(\omega) \alpha_{\mathrm{A}}^{\prime}(\omega) \omega^{-3} \mathrm{~d} \omega}{\int f_{\mathrm{D}}(\omega) \alpha_{\mathrm{A}}^{\prime \prime}(\omega) \omega^{-3} \mathrm{~d} \omega} \frac{\hbar k_{\mathrm{FRET}}}{2}
$$

The imaginary polarizability, $\alpha_{\mathrm{A}}^{\prime \prime}(\omega)$, can be extracted from an absorption spectrum and eq 9 (there is no need to worry about multiplicative constants because they cancel in the ratio). The Kramers-Kronig relation then gives $\alpha^{\prime}(\omega)$. If the Förster radius, $R_{0}$, and the lifetime of the donor are known, $k_{\text {FRET }}$ can easily be calculated from

$$
k_{\mathrm{FRET}}=\frac{1}{\tau_{\mathrm{D}}}\left(\frac{R_{0}}{r}\right)^{6}
$$

The ratio of integrals in eq 35 is typically of order 1 and is calculated for some common FRET pairs in Table 1. Figure 1 shows the emission spectrum and calculated values of $\alpha^{\prime}(\lambda)$ and $\alpha^{\prime \prime}(\lambda)$ for chlorophyll $b$. Calculations were performed by numerically integrating spectra available over the Internet. ${ }^{21}$ 
TABLE 1: Parameters for Calculating the Interaction Free Energy of Some Common FRET Pairs

\begin{tabular}{llccc}
\hline \multicolumn{1}{c}{ donor } & acceptor & $\left(2 U_{\mathrm{FRET}}\right) /\left(\hbar k_{\mathrm{FRET}}\right)^{a}$ & $\tau_{\mathrm{D}}(\mathrm{ns})$ & $R_{0}(\AA)$ \\
\hline $\mathrm{Ch} \mathrm{a}^{b}$ & $\mathrm{Ch} \mathrm{a}^{b}$ & -2.1 & & \\
$\mathrm{Ch} \mathrm{b}^{c}$ & $\mathrm{Ch} \mathrm{b}^{c}$ & -6.3 & & \\
FITC $^{d}$ & FITC $^{d}$ & -2.25 & 4.2 & 46 \\
FITC $^{d}$ & TMR $^{e}$ & 0.14 & 4.2 & 55 \\
AF594 $^{f}$ & QSY21 $^{g}$ & -0.088 & 3.9 & 77 \\
FITC $^{d}$ & AF532 $^{h}$ & 0.087 & 4.2 & 63
\end{tabular}

${ }^{a}$ The quantity $2 U_{\mathrm{FRET}} /\left(\hbar k_{\mathrm{FRET}}\right)$ is the ratio of integrals from eq 35 . ${ }^{b}$ Chlorophyll $a$ in $\mathrm{MeOH} .{ }^{c}$ Chlorophyll $b$ in diethyl ether. ${ }^{d}$ Fluorescein isothiocyanate. ${ }^{e}$ Tetramethylrhodamine. ${ }^{f}$ Alexa Fluor 594. ${ }^{g}$ Nonfluorescent quencher, diarylrhodamine derivative. ${ }^{h}$ Alexa Fluor 532.

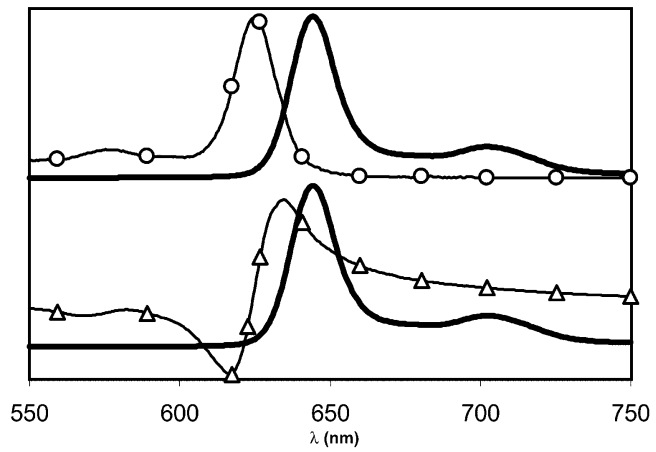

Figure 1. Spectral properties of chlorophyll $b$ in diethyl ether used for calculating the rate of homotransfer FRET (top) and the accompanying FRET force (bottom). The top panel shows (-) the emission spectrum, $f_{\mathrm{D}}(\lambda)$, and $(O)$ the absorption spectrum, $\alpha^{\prime \prime}(\lambda)$. The overlap integral yields the rate of FRET $\left(\alpha^{\prime \prime}(\lambda)\right.$ was padded with zeros for $\lambda=(700-750 \mathrm{~nm})$ to have absorption and emission spectra over comparable wavelengths). The bottom panel shows $(-)$ the same emission spectrum, $f_{\mathrm{D}}(\lambda)$, as in the top panel and $(\triangle)$ the real polarizability, $\alpha^{\prime}(\lambda)$. The overlap integral yields the interaction energy. To calculate the real polarizability, the Kramers-Kronig relations were applied to $\alpha^{\prime \prime}$ over the region $\lambda=220-750 \mathrm{~nm}$ ).

Integrations and the Kramers - Kronig calculation were carried out over the spectral window for which data was reported with no extrapolation to high or low frequencies. When the ratio of integrals (labeled in the table as $2 U_{\mathrm{FRET}} /\left(\hbar k_{\mathrm{FRET}}\right)$ ) is negative, then the FF is attractive. At a separation equal to the Förster radius, $k_{\mathrm{FRET}} \approx 1 / \tau_{\mathrm{D}}$, so $U_{\mathrm{FRET}} \approx \hbar / \tau_{\mathrm{D}}$ or the radiative line width of the donor. This miniscule shift in energy would be very difficult to detect. However, for separations typical of chlorophyll molecules in the photosynthetic antenna complex, energy transfer occurs on a time scale of $100 \mathrm{fs}$ to $1 \mathrm{ps}$, so $U_{\mathrm{FRET}} \approx$ $0.1 k_{\mathrm{B}} T$.

\section{Discussion}

How would one detect a force associated with FRET? Detection is simple if the donor and acceptor have some freedom of relative movement. The donor-acceptor separation and relative orientation both affect the dipolar coupling matrix element, $V_{\mathrm{e}}$. This matrix element in turn affects the optical properties of the dimer: it determines the rate of FRET and the Davydov splitting. A time-dependence in either of these quantities indicates movement in the excited state. Because we assume conditions under which the Förster theory is valid, the Davydov splitting is much less than the line width of donor or acceptor. However, a small shift in the maximum of the donor emission spectrum may still be detectable.

The time-dependent spectral shift associated with FRET is conceptually similar to the Stokes shift. The Stokes shift of a single fluorophore arises from a relaxation of the local environ- ment and the bonds within the molecule. ${ }^{27}$ The spectral shift associated with FRET comes from a relaxation of the medium between the two fluorophores and thus can be thought of as an intermolecular Stokes shift. Conventional Stokes shift and FRET relaxation are clearly distinct because (1) the Stokes shift is independent of the presence of a nearby fluorophore while FRET relaxation depends strongly on the intermolecular coupling and (2) the Stokes shift should occur much more quickly than FRET relaxation. For a given FRET pair and initial separation, the extent of the postexcitation spectral shift depends on the mechanical compliance of the matrix holding the molecules. Thus time-resolved FRET measurements should provide information on both the donor-acceptor spacing and the mechanical properties of the material to which they are bound.

Just as the Förster model has many extensions, so too does the force associated with it. For instance, for closely spaced molecules, it is no longer appropriate to treat the polarizability as a single tensor associated with each molecule. Rather each molecule has a nonlocal polarizability, $\alpha\left(\mathbf{r}, \mathbf{r}^{\prime}, \omega\right)$, which relates the polarization at each point $\mathbf{r}$ in the molecule to the field at all points, $\mathbf{E}\left(\mathbf{r}^{\prime}\right) .{ }^{16,28}$ Nonlocal response complicates somewhat the calculation of $V_{\mathrm{e}}$ and leads to deviation from its simple $1 / r^{3}$ dependence, but its incorporation does not create any conceptual difficulties. Once $V_{\mathrm{e}}$ has been calculated (e.g., by density functional theory), then eq 29 yields the interaction free energy.

The Förster model has also been extended to take into account time-dependent spectra of donor and acceptor. ${ }^{20}$ This extension is important when the time for energy transfer is comparable to the time for donor and acceptor to relax through their respective Stokes shifts. This extension of the theory can be readily incorporated for calculating the force. The distributions of donor and acceptor states, $g_{\mathrm{D}}\left(E_{\mathrm{D}}^{*}\right)$ and $g_{\mathrm{A}}\left(E_{\mathrm{A}}\right)$, become time-dependent quantities, but the instantaneous interaction free energy is still related to the instantaneous rate of energy transfer and the instantaneous donor and acceptor line shapes via eq 30.

Other studies of the van der Waals force with one molecule excited predict a $1 / r^{3}$ interaction potential (i.e., first order in $\left.V_{\mathrm{e}}\right) .{ }^{10-12}$ Why did our calculations not reproduce this? To get such a first-order excitonic effect requires coherent interaction of the two molecules. The Förster theory of FRET applies only in the case of very weak coupling; that is, the intermolecular coupling must be much weaker than the line width associated with coupling between each molecule and a thermal bath. Coupling to a bath destroys coherence, so the interaction potential only arises in second order in $V_{\mathrm{e}}$.

It is possible to have coherent interaction of real molecules in a bath. This coherence is the source of excitonic effects in molecular aggregates and also occurs in photosynthetic antenna complexes. When the intermolecular coupling is stronger than the coupling to the bath, the spectrum of the pair ceases to resemble the spectra of either isolated molecule. Under strong coupling, we expect that there will be a force that is first order in the coupling. Because coupling in some molecular aggregates $^{1,29}$ can be larger than $k_{\mathrm{B}} T$, we expect excitonic forces to be strong.

In summary, we have shown that an intermolecular force is intimately tied to the process of fluorescence resonance energy transfer. This force can be thought of either as optical trapping of the acceptor in the near-field of the donor or as a radiative level shift in the donor due to its damping by the acceptor. Every optical process modifies the electric field in the vicinity of the molecule in which it occurs. Thus we expect that every optical effect at finite molecular density has an associated intermolecular force. 
Acknowledgment. A.E.C thanks the members of the Bioproducts and Nanotechnology group at Cambridge for interesting discussions and acknowledges the support of the Marshall Scholarship. S.M. gratefully acknowledges the support of the Division of Chemical Sciences, Office of Basic Energy Sciences, U.S. Department of Energy.

\section{References and Notes}

(1) van Amerongen, H.; Valkunas, L.; van Grondelle, R. Photosynthetic Excitons; World Scientific: River Edge, NJ, 2000.

(2) Haas, E.; Wilchek, M.; Katchalski-Katzir, E.; Steinberg, I. Z. Proc. Natl. Acad. Sci. U.S.A. 1975, 72, 1807.

(3) Stryer, L. Апnи. Rev. Biochem. 1978, 47, 819.

(4) Weiss, S. Science 1999, 283, 1676.

(5) Freed, K. Renormalization Group Theory of Macromolecules; Wiley: New York, 1987.

(6) Chu, S.; Bjorkholm, J. E.; Ashkin, A.; Cable, A. Phys. Rev. Lett. 1986, $57,314$.

(7) Calander, N.; Willander, M. Phys. Rev. Lett. 2002, 89, 143603.

(8) Power, E. A. Introductory Quantum Electrodynamics; Longmans: London, 1964

(9) Knoester, J.; Mukamel, S. Phys. Rev. A 1989, 40, 7065. Knoester J.; Mukamel, S. Phys. Rev. A 1990, 41, 3812.

(10) Stephen, M. J. J. Chem. Phys. 1964, 40, 669

(11) Power, E. A.; Thirunamachandran, T. Phys. Rev. A 1995, 51, 3660

(12) Power, E. A.; Thirunamachandran, T. Chem. Phys. 1995, 198, 5.

(13) Yariv, A. Quantum Electronics; Wiley: New York, 1989.

(14) Novotny, L.; Hecht, B. Nano-Optics; University of Rochester: Rochester, NY, 2002 (Lecture notes on nano-optics).
(15) Mukamel, S. Principles of Nonlinear Optical Spectroscopy; Oxford University Press: New York, 1995.

(16) Tretiak, S.; Middleton, C.; Chernyak, V.; Mukamel, S. J. Phys. Chem. B 2000, 104, 4519.

(17) Davydov, A. S. Theory of Molecular Excitons; Kasha, M. Oppenheimer, M., Jr., Translators; McGraw-Hill: New York, 1962.

(18) Pantell, R.; Puthoff, H. Fundamentals of Quantum Electronics; Wiley: New York, 1969.

(19) Kubo, R. In Fluctuation Relaxation and Resonance in Magnetic Systems; Ter Haar, D., Ed.; Oliver and Boyd: Edinburgh, Scotland, 1962.

(20) Mukamel, S.; Rupasov, V. Chem. Phys. Lett. 1995, 242, 17.

(21) For chlorophyll spectra: http://omlc.ogi.edu/spectra/PhotochemCAD/html/index.html (accessed Oct 5, 2002), maintained by Oregon Medical Laser Center. For fluorophore spectra: www.probes.com (accessed Oct 5, 2002), maintained by Molecular Probes.

(22) Förster, Th. Ann. Phys. 1948, 2 (6), 55. Agranovich, V. M.; Galanin, M. D. Electronic Excitation Energy Transfer in Condensed Matter; NorthHolland: New York, 1982

(23) Langbein, D. van der Waals Attraction; Springer-Verlag: New York, 1974.

(24) Milonni, P. W. The Quantum Vacuum: An Introduction to Quantum Electrodynamics; Academic Press: New York, 1994.

(25) Power, E. A.; Zienau, S. Philos. Trans. R. Soc. London 1959, A251, 427.

(26) Andrews, D. L.; Demidov, A. A. Resonance Energy Transfer; Wiley: New York, 1999.

(27) Jimenez, R.; Fleming, G. R.; Kumar, P. V.; Maroncelli, M. Nature 1994, 369,471 .

(28) Hult, E. A.; Rydberg, H.; Lundqvist, B. Phys. Rev. A 1999, 59 , 4708. Kohn, W.; Meir, Y.; Makarov, D. E. Phys. Rev. Lett. 1998, 80, 4153.

(29) Kobayashi, T., Ed. J-Aggregates; World Scientific: River Edge, NJ, 1996 